\title{
Low-energy kaon-nucleon/nuclei interaction studies at DAФNE by AMADEUS
}

Ivana Tucaković1 ${ }^{, a}$, M. Bazzi ${ }^{1}$, C. Berucci, ${ }^{1,2}$, D. Bosnar ${ }^{3}$, A.M. Bragadireanu ${ }^{1,4}$, M. Cargnelli ${ }^{2}$, A. Clozza $^{1}$, C. Curceanu ${ }^{1}$, A. D'Uffizi ${ }^{1}$, L. Fabbietti ${ }^{5}$, C. Fiorini ${ }^{6}$, F. Ghio ${ }^{7}$, C. Guaraldo ${ }^{1}$, M. Iliescu ${ }^{1}$, T. Ishiwatari ${ }^{2}$, P. Levi Sandri ${ }^{1}$, J. Marton ${ }^{2}$, D. Pietreanu ${ }^{1,4}$, K. Piscicchia ${ }^{1,8}$, M. Poli Lener ${ }^{1}$, R. Quaglia $^{6}$, E. Sbardella ${ }^{1}$, A. Scordo ${ }^{1}$, H. Shi ${ }^{2}$, D.L. Sirghi ${ }^{1,4}$, F. Sirghi ${ }^{1,4}$, H. Tatsuno ${ }^{9}$, O. Vazquez Doce $^{5}$, E. Widmann ${ }^{2}$, and J. Zmeskal ${ }^{2}$

1 INFN, Laboratori Nazionali di Frascati, Frascati (Rome), Italy

${ }^{2}$ Stefan-Meyer-Institut für subatomare Physik, Vienna, Austria

${ }^{3}$ Physics department, University of Zagreb, Zagreb, Croatia

${ }^{4}$ Horia Hulubei National Institute of Physics and Nuclear Engineering (IFIN-HH), Magurele, Romania

${ }^{5}$ Excellence Cluster Universe, Technische Universität München, Garching, Germany

${ }^{6}$ Politecnico di Milano, Dipartimento di Elettronica, Informazione e Bioingegneria and INFN Sezione di Milano, Milano, Italy

${ }^{7}$ INFN Sezione di Roma I and Inst. Superiore di Sanitá, Rome, Italy

${ }^{8}$ Museo Storico della Fisica e Centro Studi e Ricerche "Enrico Fermi", Rome, Italy

${ }^{9}$ Department of Physics, The University of Tokyo, Tokyo, Japan

\begin{abstract}
The AMADEUS experiment deals with the investigation of the low-energy kaon-nuclei hadronic interaction at the DAФNE collider at LNF-INFN, fundamental to respond to longstanding open questions in the non-perturbative QCD in the strangeness sector. One of the most interesting aspects is to understand how hadron masses and interactions change in the nuclear environment. The antikaon-nucleon potential is investigated searching for signals from possible bound kaonic clusters, which would imply a strongly attractive antikaon-nucleon potential. AMADEUS step 0 consists in the analysis of 2004/2005 KLOE data, exploring $\mathrm{K}^{-}$absorptions in $\mathrm{H},{ }^{4} \mathrm{He},{ }^{9} \mathrm{Be}$ and ${ }^{12} \mathrm{C}$ present in setup materials. The status of the various preliminary analyses is presented, together with future perspectives.
\end{abstract}

\section{Introduction}

The AMADEUS experiment (Antikaon Matter At DAФNE: Experiments Unraveling Spectroscopy) deals with the study of the low-energy interactions of the negatively charged kaons with light nuclei. This type of studies are fundamental for understanding the non-perturbative QCD in the strangeness sector. The investigation of the antikaon-nucleon potential is done through a search of the possible bound kaonic clusters and by investigating the in-medium modifications of the $\Sigma^{*}$ and $\Lambda^{*}$ resonances properties.

\footnotetext{
a e-mail: ivana.tucakovic@lnf.infn.it
} 
The possible existence of the deeply bound kaonic nuclear states (DBKNS) was initially predicted by Wycech [1] in 1986, followed by Akaishi and Yamazaki who published their study in 2002 [2], predicting large binding energies and narrow widths for the DBKNS, as a consequence of the strongly attractive $K^{-}$p interaction. Presently ongoing various calculations using different methods are giving significantly different results for the binding energies of the kaonic nuclear states, ranging from few $\mathrm{MeV}$ up to about $100 \mathrm{MeV}$ [3-7]. To clarify the situation experimental data are needed. Until now, several experiments presented their results, but the interpretation is not yet conclusive, so the puzzle remains unsolved. Two main experimental approaches have been used in the search for the $K^{-} \mathrm{pp}$ clusters, the simplest possible DBKNS: p-p and heavy ion collisions, as, for example, in the DISTO experiment [8] and in flight or stopped $K^{-}$interactions in light nuclei, as in the FINUDA [9] and KEK-PS E549 [10] experiments. The second approach is used also in our experiment. We are looking for the $\Lambda \mathrm{p}, \Lambda \mathrm{d}$ and $\Lambda \mathrm{t}$ final states, which are the possible decay channels of the predicted DBKNS. These states give also the oportunity to study the absorption of $K^{-}$on more nucleons, processes which are still poorly known.

On the other hand, the KLOE data offers the possibility to study the properties of the $\Sigma^{*}$ and $\Lambda^{*}$ resonances and their behavior in nuclear environment. We investigate the $\Lambda(1405)$ state through its decay into $\Sigma^{0} \pi^{0}$ and $\Sigma^{+} \pi^{-}$. This state, generally accepted to be a spin $1 / 2$, isospin $\mathrm{I}=0$ and strangeness $\mathrm{S}=-1$ negative parity barion resonance $\left(J^{P}=1 / 2^{-}\right)$, assigned to the lowest $\mathrm{L}=1$ supermultiplet of the system, together to his spin-orbit partner, $\Lambda(1520)\left(J^{P}=3 / 2^{-}\right)$has yet an unknown nature. It decays only to $(\Sigma \pi)^{0}$ (I=0) through the strong interaction. The $\Lambda(1405)$ produced in $\bar{K} \mathrm{~N}$ reaction is particularly interesting due to the prediction in chiral unitary models [11-13] of two poles emerging in the scattering (with $S=-1$ and $\mathrm{I}=0$ ) in the region of the $\Lambda(1405)$ mass. One pole is located at higher energy, has narrow width, and it is mainly coupled to the $\bar{K} \mathrm{~N}$ channel, while a second, lower mass and broader pole is dominately coupled to the $\Sigma \pi$ channel [14]. Since the resonance is seen in the invariant mass spectrum of the $\Sigma \pi$ strong decay, the only chance to observe a higher mass component is to exploit the $\bar{K} N$ production mechanism, which is realized by AMADEUS. An excellent feature of AMADEUS is the possibility to study very well the $\Sigma^{0} \pi^{0}$ channel, which is free from the $I=1$ contribution and from the isospin interference.

\section{First data samples of AMADEUS}

The final AMADEUS experiment is planned to be realized by adding a dedicated setup inside the KLOE detector at the DAФNE collider at LNF-INFN in Frascati, Italy. DAФNE (Double Anular $\Phi$-factory for Nice Experiments) [15] is a double-ring $e^{-} e^{+}$collider, operating at the center of mass of the $\Phi$ meson, decaying with $\mathrm{a} \simeq 50 \%$ B.R. to almost monochromatic low momentum $\mathrm{K}^{+} \mathrm{K}^{-}$pairs $(\simeq 127 \mathrm{MeV} / \mathrm{c})$. The KLOE detector [16] is centered around the interaction region of DAФNE and consists of a large cylindrical Drift Chamber (DC), surrounded by a lead-scintillating fibers calorimeter, immersed in a magnetic field of $0.52 \mathrm{~T}$ provided by a superconduting solenoid. The drift chamber volume is filled with gas containing mainly ${ }^{4} \mathrm{He}(\simeq 90 \%)$, and a smaller amount of isobutane $\mathrm{C}_{4} \mathrm{H}_{10}$ $(\simeq 10 \%)$. The DC entrance wall is done by a $750 \mu \mathrm{m}$ layer of carbon fibre and a $150 \mu \mathrm{m}$ layer of aluminium. Monte Carlo simulations showed that about $0.1 \%$ of $K^{-}$generated by DAФNE stop in the DC volume, while 10 times more stop in the carbon entrance wall of the DC. This leads to thousands of events of $K^{-}$hadronic interactions. The KLOE detector has excellent acceptance $(\simeq 98 \%)$ and excellent resolution (e.g. for $\Lambda(1116)$ the momentum resolution is $\Delta \mathrm{p}(\mathrm{FWHM}) / \mathrm{p}=0.01$ and the invariant mass resolution is $\sigma=0.289 \pm 0.003 \mathrm{MeV} / \mathrm{c}^{2}$ ). The listed features make KLOE an ideal detector for hadronic physics studies in the strangeness sector.

The first step of AMADEUS consists in analysing the sample of about $2 \mathrm{fb}^{-1}$ of integrated luminosity of existing KLOE data (measurements performed from 2004-2005) using the detector itself 
as an active target (the gas in the volume of the DC, and the DC entrance wall). An important contribution of in-flight $K^{-}$nuclear captures is found and analysed, enabling to perform invariant mass spectroscopy of in-flight $K^{-}$nuclear capture processes. To increase the statistics, we performed a dedicated one-month run in 2012 with a pure half cylindrical carbon target, implemented in the central region of the KLOE detector, between the beam pipe and the DC wall, providing a high statistics sample of at-rest $K^{-}$nuclear interactions. The geometry of the target was optimized to stop the kaons, and had a thickness varying from $4-6 \mathrm{~mm}$ taking into account the $\Phi$ boost derived from the crossing angle of the $e^{+} e^{-}$beams at DAФNE. The carbon target insertion into the KLOE setup is shown in Fig. 1 . The total integrated luminosity of the data collected with this target was $\simeq 90 p b^{-1}$. Presently, we are proceeding with the data reconstruction and calibration proceedures.

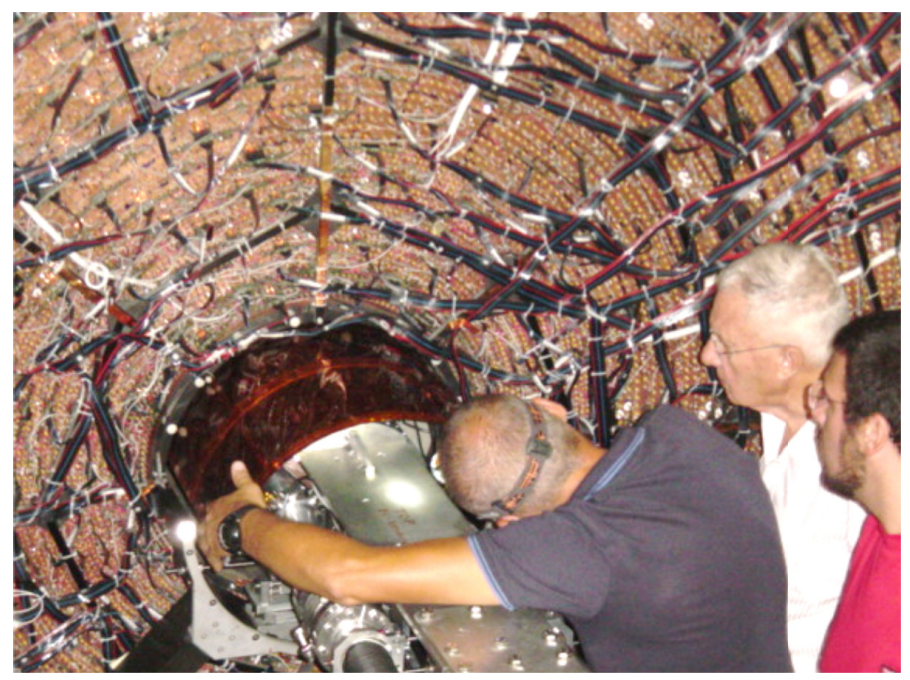

Figure 1. The insertion of the graphite target used by AMADEUS in the 2012 run inside of KLOE setup.

\section{Particle identification and event selection}

\subsection{The $\Lambda(1116)$ selection}

The presence of a $\Lambda(1116)$ represents the signature of a $K^{-}$hadronic interaction. The $\Lambda(1116)$ was found through the reconstruction of its decay into $\Lambda \rightarrow p+\pi^{-}(B R=63.9 \pm 0.5) \%$. The protons and pions selection and identification is done using the $\mathrm{dE} / \mathrm{dx}$ information, measured with the truncated mean of ADC counts from the wires hit in the DC and the momentum of the track, as it is shown in Fig. 2.

The invariant mass $m_{p \pi^{-}}$is shown in Fig. 3. In order to separate the two samples of $K^{-}$absorption processes occuring in the gas filling the DC volume and in the DC entrance wall, we performed cuts on the radial position of the $\Lambda$ decay vertex based on MC simulations.

For the $\Lambda-\mathrm{p} / \mathrm{d} / \mathrm{t}$ channels study, once the $\Lambda$ has been identified, it is extrapolated back along its expected decay path and an additional charged track, coming from the same vertex, is searched for. The procedure is similar to the one explained before. 


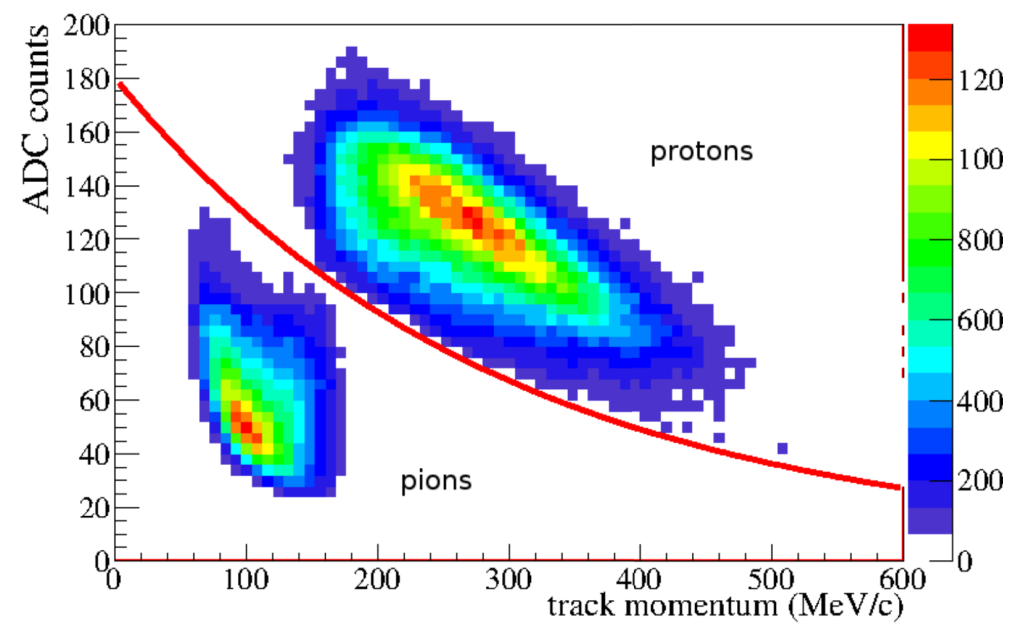

Figure 2. The $d E / d x$ (in ADC counts) vs. momentum for the selected proton (up) and pion (down) tracks in the final selection. The selection function is displayed in red.

\subsection{The $\Sigma^{0} \pi^{0}$ events identification}

To select the $\Sigma^{0} \pi^{0}$ events, along with the $\Lambda(1116)$ identification, a search for three additional in-time photon clusters has to be done, according to the reaction:

$$
K^{-} p \rightarrow \Sigma^{0} \pi^{0} \rightarrow\left(\Lambda \gamma_{3}\right)\left(\gamma_{1} \gamma_{2}\right) \rightarrow\left(p \pi^{-}\right) \gamma_{1} \gamma_{2} \gamma_{3}
$$

where $\gamma_{3}$ represents the photon coming from the $\Sigma^{0}$ decay, while $\gamma_{1}$ and $\gamma_{2}$ are the photons from the $\pi^{0}$ decay.

A pseudo-chisquare minimization is performed, searching for three neutral clusters in the calorimeter $\left(E_{c l}>20 \mathrm{MeV}\right)$, in time from the decay vertex position of the $\Lambda(1116)\left(\mathbf{r}_{\Lambda}\right)\left(\chi_{t}^{2}=\right.$ $\left(t_{i}-t_{j}\right)^{2} / \sigma_{t}^{2}$ where $t_{i}$ is the $\mathrm{i}$ - $t$ cluster time subtracted by the time of flight in the speed of light hypothesis). According to dedicated MC simulations a cut was optimized on this variable as $\chi_{t}^{2} \leq 20$.

Once the three candidate photon clusters are chosen, their assignment to the correct triplet of photons, $\left(\gamma_{1}, \gamma_{2}, \gamma_{3}\right)$, is based on a second pseudo-chisquare minimization $\left(\chi_{\pi \Sigma}^{2}\right) \cdot \chi_{\pi \Sigma}^{2}$ involves both the $\pi^{0}$ and $\Sigma^{0}$ masses. $\chi_{\pi \Sigma}^{2}$ is calculated for each possible combination and the minimizing triplet is selected. The $\chi_{\pi \Sigma}^{2} \leq 45$ cut was optimized based on MC simulations.

According to true MC information the algorithm has an efficiency of $(98 \pm 1) \%$ in recognizing photon clusters and an efficiency of $(78 \pm 1) \%$ in distinguishing the correct $\gamma_{1} \gamma_{2}$ pair $\left(\pi^{0}\right.$ decay) from $\gamma_{3}$.

A check is performed on the clusters energy and distance to avoid the selection of splitted clusters (single clusters in the calorimeter erroneously recognized as two clusters) for $\pi^{0} \mathrm{~s}$. Cluster splitting is found to not affect significantly the sample.

In Fig. 4 is shown the obtained invariant mass $m_{\Lambda \gamma 3}$ (for absorptions in the gas) together with a Gaussian fit. The resolution in the $m_{\Lambda \gamma 3}$ invariant mass is $\sigma_{m_{\Lambda \gamma 3}} \sim 15 \mathrm{MeV} / \mathrm{c}^{2}$. The resolutions on $\rho_{\Lambda}$ for the final selected $\Lambda \mathrm{s}$ are $\sigma_{\rho_{\Lambda}} \sim 0.20 \mathrm{~cm}$ (DC wall) and $\sigma_{\rho_{\Lambda}} \sim 0.13 \mathrm{~cm}$ (DC gas). The resolutions on the $\Lambda$ momentum are $\sigma_{p_{\Lambda}} \sim 4.5 \mathrm{MeV} / \mathrm{c}$ (DC wall) and $\sigma_{p_{\Lambda}} \sim 1.9 \mathrm{MeV} / \mathrm{c}$ (DC gas). Each quoted 


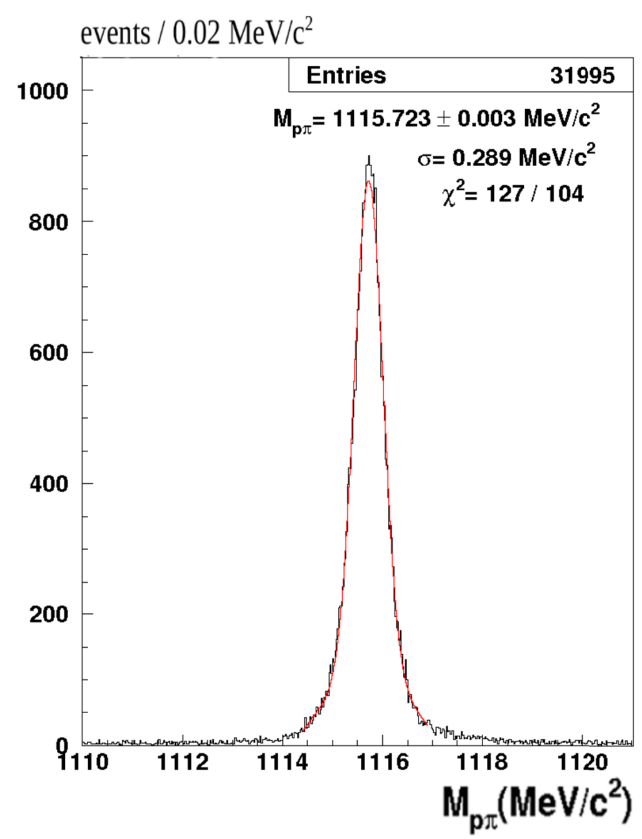

Figure 3. The invariant mass spectrum for the pion-proton pairs.

mean resolution corresponds to Gaussian fits to the distributions of the originally generated true-MC quantities subtracted by the reconstructed ones. The better resolution for the measured variables corresponding to $\mathrm{K}^{-}$hadronic interactions in the gas filling the KLOE DC is a consequence of the charged particles energy loss, mainly in the material of the DC entrance wall, particularly important for protons.

\section{Status of the analyses}

This section gives an overview of the preliminary results obtained in various channels where different level of analysis is achieved until now in our endeavour to investigate the low energy interactions of negatively charged kaons with nuclear matter.

The study of the correlations between $\Lambda \mathrm{p}, \Lambda \mathrm{d}$ and $\Lambda \mathrm{t}$ pairs consists of invariant mass spectra, momenta and angular distributions investigations, in order to explore the various physical processes, such as the multi-nucleon absorption of $K^{-}, \Sigma$ to $\Lambda$ internal conversion, all the way to the possible formation of deeply bound kaonic nuclear clusters. As an example in Fig. 5, we show the $\Lambda$ p invariant mass spectrum obtained. The blue curve represents all the reconstructed $\Lambda \mathrm{p}$ events, while the red one represents events where a negatively charged pion is found in the final state too. The presence of pion is a characteristic signal of $K^{-}$absorption on one nucleon. The MC studies are ongoing in attempts to fit the obtained spectra, especially in the $\Lambda \mathrm{p}$ channel where we have the possibility to extract quantitative information, such as single-over-double nuclear absorption rate per stopped $\mathrm{K}^{-}$in ${ }^{4} \mathrm{He}$ and the $\Sigma$ to $\Lambda$ conversion rate.

We are performing the search for the $\Lambda(1405)$ through its decay in $\Sigma^{0} \pi^{0}$ (which is purely isospin 0 ) and $\Sigma^{+} \pi^{-}$(the analysis of the $\Sigma^{-} \pi^{+}$decay channel started recently with a characterization of neutron 


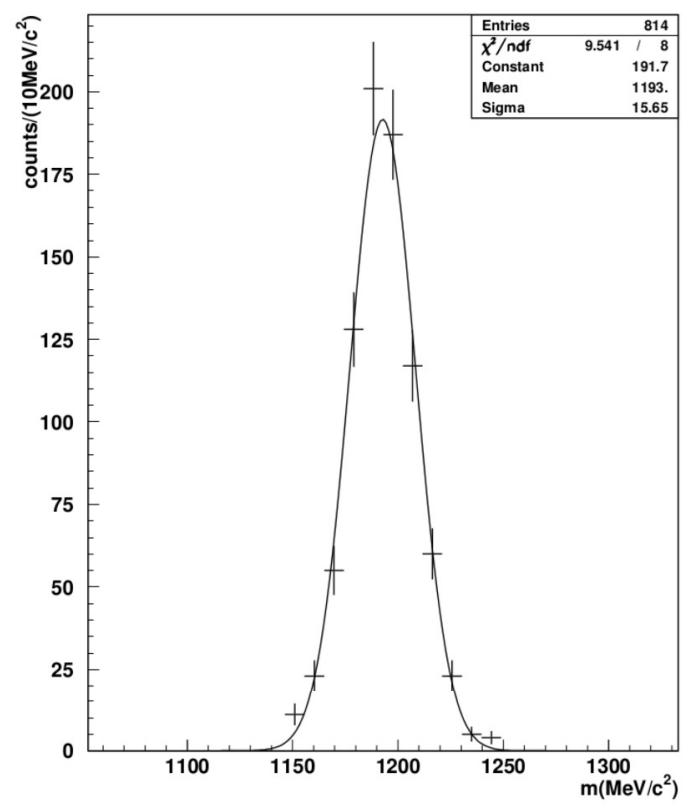

Figure 4. $m_{\Lambda \gamma 3}$ invariant mass distribution, together with a Gaussian fit.

clusters in the KLOE calorimeter). The line shapes of the three combinations $(\Sigma \pi)^{0}$ were recently obtained, for the first time, in a single experiment by [17] and were found to be different. Of extreme interest will be a precise measurement of the $\frac{\Sigma^{+} \pi^{-}}{\Sigma^{-} \pi^{+}}$production ratio in different targets, with strong consequences on the nature of the $\Lambda^{*}$ state $[18,19]$.

We are also exploring the $\Lambda \pi^{-}$(isospin $\mathrm{I}=1$ ) production, both from direct formation process and from internal conversion of a primary produced $\Sigma$ hyperon $\left(\Sigma N \rightarrow \Lambda N^{\prime}\right)$. Given the excellent resolution for the $\Lambda \pi^{-}$invariant mass, we are aiming to distinguish resonant $\left(\Sigma^{*-}\right)$ from non resonant formation, extracting, for the first time, the ratio between the transition amplitudes module for the two processes.

\section{Conclusions and future perspectives}

In this paper an overview of the ongoing studies in the AMADEUS framework are presented, both for the 2004-2005 KLOE data, and for the dedicated carbon-target data collected in 2012. We demonstrated that the KLOE detector together with the unique features of the DAФNE collider, gives the possibility to perform high quality physics in order to produce new constraints on the existing theoretical models in the non-perturbative QCD in the strangeness sector. 


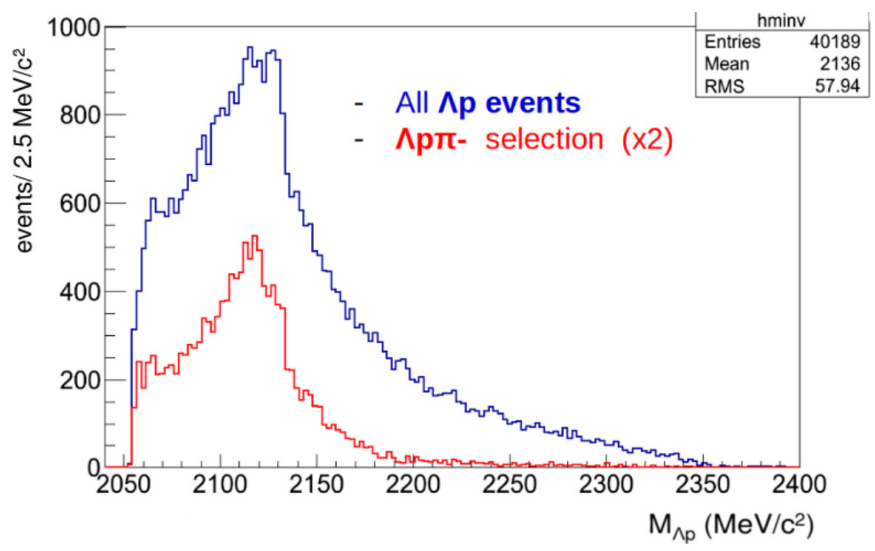

Figure 5. The $\Lambda p$ invariant mass spectrum: blue curve - all the $\Lambda p$ pairs, red curve $-\Lambda p \pi^{-}$events.

In parallel to the ongoing data analysis, from the experimental point of view we are considering the construction of a more refined dedicated setup (inner tracker and trigger system), to explore in detail and with a higher precision the low-energy kaons interactions processes with targets going from hydrogen and helium, to lithium and beryllium.

\section{Acknowledgements}

We thank F. Bossi, S. Miscetti, E. De Lucia, A. Di Domenico, A. De Santis and V. Patera for the guidance in performing the analyses, and all the KLOE Collaboration and DAФNE staff for the fruitful collaboration. Thanks to Doris Stueckler and Leopold Stohwasser for the technical realization of the carbon-target.

Part of this work was supported by the European Community-Research Infrastructure Integrating Activity "Study of Strongly Interacting Matter" (HadronPhysics2, Grant Agreement No. 227431, and HadronPhysics3 (HP3) Contract No. 283286) under the EU Seventh Framework Programme.

\section{References}

[1] S. Wycech, Nucl. Phys. A 450, 399c-402c (1986)

[2] Y. Akaishi, T. Yamazaki, Phys. Rev. C, 65, 044005 (2002)

[3] Y. Akaishi, T. Yamazaki, Phys. Lett. B, 535, 70 (2002)

[4] Dote, Hyodo, Weise, Phys. Rev. C, 79, 014003 (2009)

[5] Shevchenko, Gal, Mares, Phys. Rev. Lett. 98, 082301 (2007)

[6] Ikeda, Sato, Phys. Rev. C, 79, 035201 (2009)

[7] Wycech, Green, Phys. Rev. C 79, 014001 (2009)

[8] M. Maggiora et al., Nucl. Phys. A 835, 43-50 (2010)

[9] M. Agnello et al., Phys. Rew. Lett.94, 212303 (2005)

[10] T. Suzuki et al., Mod. Phys. Lett. A 23, 2520-2523 (2008)

[11] N. Kaiser, B. P. Siegel, W. Weise, Nuclear Phys A594, 325 (1995)

[12] E. Oset, A. Ramos, Nuclear Phys. A635, 99 (1998)

[13] J. A. Oller, U. G. Meissner, Phys. Lett. B500, 263 (2001) 
[14] Y. Ikeda, T. Hyodo, W. Weise, Nucl. Phys. A881, 98 (2012)

[15] R. Baldini et al., Report LNF-90/031, (R) (1990)

[16] F. Bossi, E. De Lucia, J. Lee-Franzini, S. Miscetti, M. Palutan and KLOE coll., Riv. Nuovo Cim., 31, 531-623 (2008)

[17] K. Moriya et al. (CLAS Collaboration), Phys. Rev. C 87, 035206 (2013)

[18] L. R. Staronski, S. Wycech, Nucl. Phys. 131361 (1987)

[19] A. Ohnishi et al., Phys. Rev. C 5652767 (1997) 